\title{
Presencia de caries dental en pacientes con labio y paladar hendido: una revisión sistemática
}

\author{
Presence of dental caries in patients with cleft lip and palate: a systematic review
}

Daniela Barrero Lizcano', María Alejandra Pinto Pabón', Luisa Teresa Flórez Meneses²

Para citar este artículo: Barrero-Lizcano D, Pinto-Pabón MA, Flórez LT. Presencia de caries dental en pacientes con labio y paladar hendido: una revisión sistemática. Revista UstaSalud 2016;15:27-35.

Licencia Creative Commons

\section{(c) (1) (\$) $\Theta$} lo tanto, los lectores pueden acceder libremente a los artículos en su formato .pdf, igualmente podrán descargarlos y difundirlos; sin embargo no podrán modificarlos o alterarlos, adicionalmente se debe reconocer la autoría de las personas que figuran en las publicaciones, pero estas no podrán comercializadas.

\section{RESUMEN}

Objetivo: realizar revisión sistemática de estudios publicados sobre la presencia, susceptibilidad y prevalencia de caries dental en pacientes con labio y paladar hendido (LPH) y en individuos sin esta condición.

Materiales y métodos: se realizó una búsqueda en tres bases de datos (Pubmed, Web of Science, Scopus) para identificar artículos con estudios de casos y controles que reportaran la presencia de caries dental en pacientes con y sin este evento. Del estudio hicieron parte artículos texto completo y publicados entre el 2002 y el segundo período del 2015.

Resultados: en la revisión se evaluaron 12 publicaciones de casos y controles, se encontró que diez de estos artículos concluyen que los pacientes con LPH, tienen mayor susceptibilidad de desarrollar caries dental en contraste con los pacientes controles, de igual manera se observaron diferencias estadísticamente significativas para varios estudios.

Conclusión: puede decirse que los individuos con esta condición están en mayor riesgo de desarrollar lesiones cariosas, puesto que aquellos sometidos a cirugía de LPH, por ejemplo, podrían tener miedo y dolor al momento de realizar la higiene bucal por las características del tejido cicatrizal, entre otras razones.

Palabras claves: Labio y paladar hendido; caries dental, revisión sistemática.

\section{ABSTRACT}

Objective: to carry out a systematic review of published studies regarding the presence, susceptibility and prevalence of dental caries in patients with cleft lip and palate (CLP) and individuals without this condition, as well.

Materials and methods: three databases (Pubmed, Web of Science, Scopus) were chosen for the electronic search, and only case control studies reporting the presence of dental caries in patients with and without this event were considered for this paper. The researchers searched for full text papers published between 2002 and mid 2015.

Results: in this systematic review, 12 case controls studies were assessed, it was found that ten of these articles conclude that patients with LPH are more susceptible to develop dental caries in contrast to the control patients, in the same way statistically significant differences were observed for some of the studies.

Conclusion: the studies reviewed suggest that individuals with this condition are at higher risk of developing carious lesions since those undergoing CLP surgery, for example, could be afraid and feel pain when performing oral hygiene due to characteristics of the scar tissue and other reasons.

Keywords: Cleft lip and palate; dental caries, systematic review.
${ }^{1}$ Facultad de Odontología, Universidad Santo Tomás, Bucaramanga

${ }^{2}$ Grupo de Investigación Salud Integral Bucal (SIB), Facultad de Odontología, Universidad Santo Tomás, Bucaramanga

Autor de correspondencia:

Luisa Teresa Flórez Meneses

Correo electrónico:

luisa.florez@ustabuca.edu.co
Recibido para publicación:

22 de marzo de 2016.

Aceptado para publicación:

29 de julio de 2016. 


\section{INTRODUCCIÓN}

Las condiciones de labio y paladar hendido (LPH) son unos de los defectos congénitos más comunes en el mundo, debido a que constituye el $65 \%$ de las anomalías que afectan cabeza y cuello ${ }^{1}$. A nivel mundial se ha reportado una prevalencia de 0,2 a 2,7 por cada 1000 nacimientos, mientras que en Colombia se ha reportado que podría afectar desde 1 en 500 a 1 en 1000 individuos ${ }^{2}$.

Esta patología se produce entre la sexta y décima semana de vida intrauterina y se desarrolla en la etapa temprana del embarazo ${ }^{3}$, se produce cuando no existe una adecuada fusión de los labios ni del paladar; un niño puede tener labio hendido, paladar hendido o ambas condiciones ${ }^{4}$.

Existen varios factores que aumentan el riesgo de presentar LPH, siendo el más común, el factor genético de los padres ${ }^{5}$, incluyendo también los cuidados inadecuados de la madre durante el embarazo como la ingesta de alcohol y tabaco ${ }^{6}$. No obstante, el uso de algunos medicamentos como las benzodiacepinas ${ }^{7}$ aumentan la posibilidad de que el niño nazca con esta condición ${ }^{8,9}$.

El factor de crecimiento transformante alfa ( $T G F a$ Gen) es el que se encuentra asociado con el crecimiento craneofacial ${ }^{10}$ y estudios determinaron que los genes que se encuentran directamente relacionados con LPH son $\mathrm{N}$-acetiltransferasas $(N A T)^{11}$, receptor $\mathrm{A}$ de ácido retinoico $(R A R A)$, factor de crecimiento transformante beta 3 (TGF 33$)$ y homeobox-MSH (MSX1 genes) ${ }^{12}$.

La higiene bucal de los pacientes que presentan labio y paladar hendido es fundamental, debido a que por medio de la cavidad oral se realizan funciones como la fonación, la respiración, la digestión; si no se tiene una limpieza óptima y adecuada se genera un ambiente propicio para producir patologías como la caries dental, que es una enfermedad caracterizada por el desequilibrio entre la superficie dental y los fluidos de la placa circundante en la que existen tres factores principales ${ }^{13}$ : el huésped (higiene bucal, la saliva y los dientes), la microbiota (infecciones bacterianas) y el sustrato (dieta cariogénica) ${ }^{14,15}$. Además de estos factores, se debe tener en cuenta también, el tiempo para que se dé la formación de la caries den- tal; asimismo, estos pacientes por lo general presentan apiñamiento dental, aparatos ortodónticos y en algunos casos maloclusiones que aumentan la susceptibilidad a la caries dental.

La recopilación de artículos científicos de casos y controles que evalúen si la presencia de caries dental en pacientes con LPH es mayor en comparación con los que no presentan esta condición puede dar una idea más concluyente a este aspecto, por tanto, el objetivo de este estudio fue realizar una revisión sistemática que incluyera únicamente estudios de casos y controles, los cuales determinaban la presencia de caries dental en pacientes con y sin LPH, además de describir otros aspectos relacionados con la salud bucal y la prevalencia de caries en los participantes de los estudios.

\section{MATERIALES Y MÉTODOS}

Se hizo una revisión sistemática cuyo fin fue extraer información existente de estudios previos, con el objeto de sintetizar los datos disponibles, se buscaron artículos de casos y controles en las bases de datos: Scopus, Pubmed y Web Of Science que estuvieran publicados entre el 2002 y el segundo período del 2015. Este estudio se desarrolló bajo los criterios de la declaración PRISMA ${ }^{16}$ para revisiones sistemáticas y meta-análisis.

Se decidió incluir artículos en texto completo, en inglés, y además se tuvo en cuenta que el diseño del estudio fuera de casos y controles; el objetivo de los estudios debía enfocarse en determinar cuál de los dos grupos (individuos con y sin $\mathrm{LPH}$ ) podría tener más frecuencia de caries dental. Al momento de realizar la búsqueda en las tres bases de datos, se usaron los siguientes términos MeSH (Cleft palate, cleft lip, Dental caries), utilizándolos de manera individual y combinándolos entre sí con el objeto de alcanzar la mayor cantidad de artículos publicados. Para la realización de la estrategia de búsqueda, se utilizó el conector universal "AND".

- Cleft lip

- Cleft palate

- Dental caries

- (“Cleft Lip"[Mesh]) AND “Dental Caries”[Mesh]

- (“Cleft palate"[Mesh]) AND "Dental Caries"[Mesh]

Se seleccionaron artículos de las bases de datos (Pubmed, Web Of Science, Scoupus) relacionados con 
la presencia de caries dental en pacientes con labio y paladar hendido. Posteriormente se realizó la extracción de datos y se plasmó la información en una hoja de cálculo de $\mathrm{Excel}^{17}$, en la cual se registraron aspectos como el año de publicación, primer autor, objetivo del estudio, resultados y conclusiones, con el fin de poder realizar el análisis cualitativo de los datos y de esta manera determinar si efectivamente los niños con LPH son más susceptibles a presentar caries dental con respecto a los niños que no presentan hendiduras bucales.

Dos investigadores (DBL Y MPP) realizaron la búsqueda y la selección de los artículos, mientras que el tercer investigador (LTFM) intervino en aquellos casos de disparidad; entonces, en consenso se determinó cuales artículos debían ser incluidos en el trabajo de investigación y cuáles no.

\section{RESULTADOS}

Se inició con la identificación de los artículos $(n=372)$, posteriormente se leyeron los títulos y los resúmenes para determinar si se incluían o no en el trabajo, no obstante, se encontraron 39 duplicados que fueron in- mediatamente retirados; 313 fueron excluidos porque no cumplían con los criterios de elegibilidad, por lo tanto, se leyeron 20 artículos en texto completo y después de leerlos por los tres investigadores, se identificó que ocho de ellos tenían otras variables dependientes dentro de sus objetivos y otros realmente no seguían un diseño de casos y controles. Finalmente, se incluyeron 12 estudios de casos y controles en la revisión sistemática (Figura 1).

Es importante mencionar que de los 12 artículos escogidos 5 de ellos fueron publicados en la revista: Cleft Palate-Craniofacial Journal y correspondían a investigaciones de países como China, Brasil y Siria, entre otros. Los artículos se publicaron desde el 2004 al 2015 y evaluaron participantes de diferentes rangos de edad (desde 1 año hasta 28 años). Así mismo, las investigaciones incluyeron personas tanto del género femenino como masculino, y en siete de ellos el grupo de hombres fue más numeroso (Tabla 1).

En los estudios encontrados, los diagnósticos de caries se realizaron de acuerdo con el International Caries Detection And Assessment system (ICDAS), otros prefirieron usar el índice de Dientes Cariados Obturados

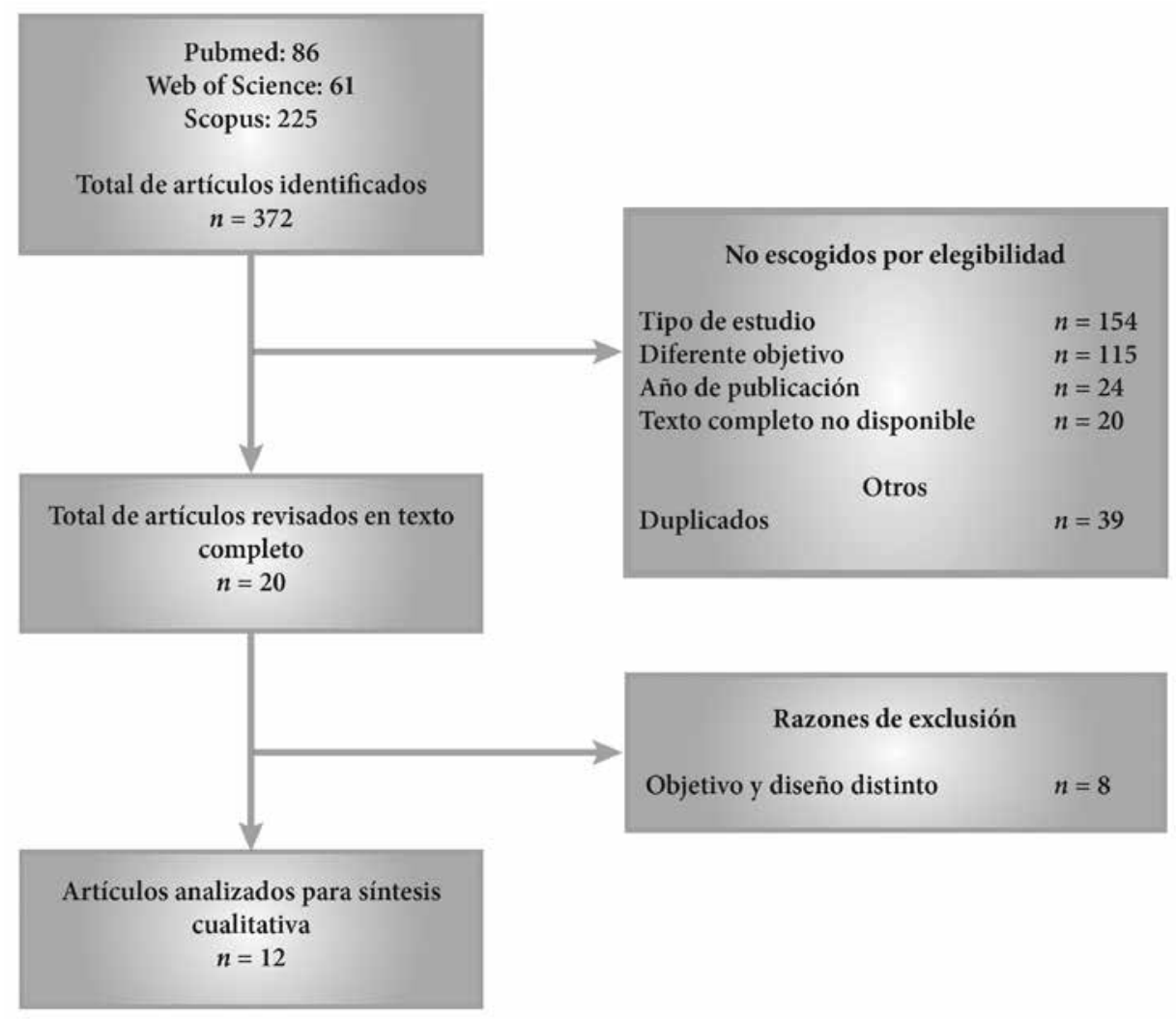

Figura 1. Flujograma de las diferentes fases de la revisión sistemática 
y Perdidos (COP) conocido por sus siglas en inglés De igual forma, algunos artículos reportaron el índicomo DMFT (Decayed/Missing/Filled Teeth) para ce DMFS que hace referencia al índice por superficie dientes permanentes y dfmt para dientes temporales. dental (Tabla 2).

Tabla 1. Descripción general de los artículos

\begin{tabular}{|c|c|c|c|c|c|c|c|}
\hline \multirow{2}{*}{$\begin{array}{l}\text { Artículo } \\
\text { Sundell y cols. } 2015^{18}\end{array}$} & \multirow{2}{*}{$\begin{array}{l}\text { Revista } \\
\text { Acta Odontológica Scandinavica. }\end{array}$} & \multirow{2}{*}{$\begin{array}{l}\text { País } \\
\text { Suecia }\end{array}$} & \multicolumn{2}{|c|}{ Participantes } & \multirow{2}{*}{$\frac{\text { Rango de edad }}{5-10 \text { años }}$} & \multirow{2}{*}{$\begin{array}{c}\begin{array}{c}\text { Número de } \\
\text { casos }\end{array} \\
139\end{array}$} & \multirow{2}{*}{$\begin{array}{c}\begin{array}{c}\text { Número de } \\
\text { controles }\end{array} \\
313\end{array}$} \\
\hline & & & $\delta: 228$ & $+: 224$ & & & \\
\hline Sundell y cols. $2015^{19}$ & BMC Oral Health & Suecia & $0^{1}: 214$ & $q: 216$ & $5-10$ años & 133 & 297 \\
\hline Kirchberg y cols. $2014^{20}$ & The Cleft Palate-Craniofacial Journal & Alemania & $\delta^{\pi}: 430$ & q:413 & $1-6$ años & 295 & 548 \\
\hline Chopra y cols. $2014^{21}$ & Journal of Medical Sciences & India & ठิ: 57 & $+: 39$ & $4-6$ años & 48 & 48 \\
\hline Pisek y cols. $2014^{22}$ & J Med Assoc Thai & Tailandia & J: 82 & ○: 104 & $10-14$ años & 68 & 118 \\
\hline King y cols. $2013^{23}$ & The Cleft Palate-Craniofacial Journal & China & - & - & 2 - 7 años & 132 & 132 \\
\hline Freitas y cols. $2013^{24}$ & The Cleft Palate-Craniofacial Journal & Brasil & $\delta: 22$ & q: 38 & $12-21$ años & 30 & 30 \\
\hline Tannure y cols. $2012^{25}$ & Pediatr Dent & Brasil & & - & $4-21$ años & 115 & 230 \\
\hline Zhu y cols. $2010^{26}$ & Cleft Palate-Craniofacial Journal, & China & స: 465 & O: 254 & $3-25$ años & 380 & 339 \\
\hline Mutarai y cols. $2008^{27}$ & Cleft Palate Craniofac J & Tailandia & O':74 & q 64 & 18 - 36 meses & 69 & 69 \\
\hline Al-Wahadni y cols. $2005^{28}$ & Cleft Palate-Craniofacial Journal & Jordania & $\delta: 36$ & P $: 28$ & $10-28$ años & 32 & 32 \\
\hline Ahluwalia y cols. $2004^{29}$ & J Dent Res & Londres & - & - & $6-16$ años & 81 & 61 \\
\hline
\end{tabular}

ㅇ (femenino), ${ }^{\Uparrow}$ (masculino)

Tabla 2. Resultados de los artículos en relación con el índice de caries

\begin{tabular}{|c|c|c|c|c|c|}
\hline Artículo & Edad de los participantes & Índice de caries & $\begin{array}{c}\text { Promedio casos } \\
\text { X (DE) }\end{array}$ & $\begin{array}{c}\text { Promedio controles } \\
\text { X(DE) }\end{array}$ & $\begin{array}{c}\text { Valor de p o medida de } \\
\text { asociación }\end{array}$ \\
\hline \multirow[t]{3}{*}{ Sundell y cols. ${ }^{18}$} & 5 años & Dmfs & $1,2 \pm 2,6$ & $0,9 \pm 3,2$ & $0,01^{\star} \mathrm{OR}=1,89$ \\
\hline & 10 años & Dmfs & $0,9 \pm 1,5$ & $1,2 \pm 2,6$ & $>0,05^{\star}$ \\
\hline & & DMFS & $0,7 \pm 1,4$ & $0,5 \pm 1,5$ & $>0,05^{*}$ \\
\hline \multirow[t]{2}{*}{ Sundell y cols. ${ }^{19}$} & 5 años & Dmfs & $1,4 \pm 2,9$ & $1,4 \pm 2,9$ & - \\
\hline & 10 años & dmfs+DMFS & $1,8 \pm 2,5$ & $3,5 \pm 4,5$ & - \\
\hline Kirchberg y cols..$^{20}$ & 1-6 años & Dmft & $2,0 \pm 3,2$ & $1,24 \pm 2,5$ & $0,001^{*}$ \\
\hline Chopra y cols. ${ }^{21}$ & 4-6 años & $\mathrm{Dmft}$ & $3,8 \pm 4,5$ & $2,0 \pm 2,5$ & $0,04^{*}$ \\
\hline \multirow[t]{2}{*}{ Pisek y cols. ${ }^{22}$} & $10-14$ años & Dmft & $0,7 \pm 1,4$ & $1,1 \pm 1,7$ & $0,08^{*}$ \\
\hline & & DMFT & $0,8 \pm 1,2$ & $0,4 \pm 0,9$ & $<0,01^{*}$ \\
\hline \multirow[t]{2}{*}{ King y cols..$^{23}$} & 2-4 años & Dmft & $1,5 \pm 2,9$ & $1,6 \pm 2,8$ & $0,754^{*}$ \\
\hline & 5-7 años & Dmft & $5,2 \pm 4,4$ & $2,9 \pm 3,9$ & $0,0006^{*}$ \\
\hline \multirow[t]{2}{*}{ Freitas y cols. ${ }^{24}$} & 12- 21 años & DMFT & $8,2 \pm 4,5$ & $7,2 \pm 5,3$ & $0,498^{*}$ \\
\hline & & DMFS & $10,8 \pm 6,6$ & $8,8 \pm 8,2$ & $0,356^{*}$ \\
\hline \multirow[t]{2}{*}{ Tannure y cols. ${ }^{25}$} & 4-21 años & DMFT & $1,20 \pm 1,8$ & $0,9 \pm 1,8$ & $0,16^{*}$ \\
\hline & & $\mathrm{Dmft}$ & $1,7 \pm 2,1$ & $2,6 \pm 2,9$ & $0,02^{*}$ \\
\hline \multirow[t]{3}{*}{ Zhu y cols. ${ }^{26}$} & 3-5 años & $\mathrm{dmft}+\mathrm{DMFT}$ & $2,5 \pm 4,0$ & $1,9 \pm 2,0$ & $0,953^{*}$ \\
\hline & 6-12 años & $\mathrm{dmft}+\mathrm{DMFT}$ & $4,2 \pm 3,7$ & $3,1 \pm 2,9$ & $0,019^{*}$ \\
\hline & 13-25 años & $\mathrm{dmft}+\mathrm{DMFT}$ & $2,4 \pm 2,6$ & $1,4 \pm 2,0$ & $<0,01^{*}$ \\
\hline Mutarai y cols. ${ }^{27}$ & $18-36$ meses & Dmft & $9,2 \pm 6,0$ & $6,5 \pm 4,5$ & $<0,01^{*}$ \\
\hline \multirow[t]{2}{*}{ Al-Wahadni y cols. ${ }^{28}$} & $10-15$ años & DMFT & $4,8 \pm 5,1$ & $2,1 \pm 1,1$ & $0,04^{*}$ \\
\hline & 16-28 años & DMFT & $5,4 \pm 5,9$ & $2,1 \pm 1,1$ & $0,08^{*}$ \\
\hline \multirow[t]{2}{*}{ Ahluwalia y cols. ${ }^{29}$} & 6-16 años & Dmft & $2,4 \pm 0,3$ & $0,6 \pm 0,2$ & $<0,001^{*}$ \\
\hline & & DMFT & $1,56 \pm 0,2$ & $0,5 \pm 0,2$ & $<0,001^{*}$ \\
\hline
\end{tabular}

DFMT (Cariado-Obturado-Perdido en dentición permanente), dfmt (Cariado-Obturado-Perdido en dentición temporal), DFMs (Cariado-Obtura do-Perdido en dentición permanente por superficie), dfmts (Cariado-Obturado-Perdido en dentición temporal por superficie). ${ }^{\star P r u e b a ~ U ~ d e ~ M a n n-W h i t n e y . ~}$ 
Los promedios respecto al índice COP oscilaron entre 0,5 a 9,2; es importante destacar que en 17 de los 23 índices encontrados, los casos (pacientes con LPH) presentaron mayor número de dientes cariados. Investigaciones como las de Sundell y cols. ${ }^{18,19}$ evidenciaron que a la edad de 10 años en la dentición temporal y mixta podrían, de hecho, los controles presentar mayor número de dientes con caries ( $>0,05)$ y a los cinco años de edad podrían estar en igualdad de condiciones; adicionalmente otros autores ${ }^{22,23,25}$ encontraron tendencias similares, donde los controles tuvieron índices superiores, entre ellos solo uno de latinoamerica ${ }^{25}$.

Varios estudios ${ }^{18,20-29}$ encontraron diferencias estadísticamente significativas entre los grupos (pacientes con LPH y sin LPH) al momento de comparar sus promedios de caries según el índice COP.

Únicamente 5 artículos reportaron la prevalencia de caries de la población en porcentaje, por ejemplo, Pisek y colaboradores. ${ }^{22}$ determinaron que el $48,5 \%$ de los casos presentaron caries dental en comparación con el $20,3 \%$ de los controles con un valor de $\mathrm{p}<0,01$ (tabla 3); este valor se halló utilizando la prueba estadística de Chi-cuadrado. Los únicos autores que no encontraron una diferencia estadísticamente significativa entre los grupos fueron Sundell y colaboradores. ${ }^{18}$ en el grupo de participantes de 10 años ( $\left.\mathrm{p}>0,05\right)$; Chopra y colaboradores ${ }^{21}$ que hallaron un valor de $\mathrm{p}=0,07$ y Mutarai y colaboradores. ${ }^{22}$ que reportaron una prevalencia alta de caries en ambos grupos y un valor de $\mathrm{p}=1,00$ (Tabla 3 ).

No obstante, los artículos también reportaron información de otros parámetros evaluados (Tabla 4), como por ejemplo:

Defectos de desarrollo del esmalte. Sundell y colaboradore ${ }^{18}$ evaluaron este parámetro y reportaron que entre los 5 años de edad, la prevalencia de hipomineralización / hipoplasia fue del $61 \%$ en el grupo de participantes con LPH comparado con un $6 \%$ en el grupo control, hallándose una diferencia significativa $\mathrm{p}<0,05$. En general el promedio de hipomineralización/hipoplasia fue superior $(3,0)$ en los participantes con LPH con respecto al grupo control $(1,8)(\mathrm{p}<0,01)$.

Presencia de lactobacilos y estreptococos. En este parámetro Sundell y colaboradores ${ }^{19}$ mostraron que
81 de los participantes con LPH presentaban una baja presencia de lactobacilos en comparación a 91 individuos del grupo control, encontrando una diferencia estadísticamente significativa $(\mathrm{p}=0,001)$.

Ahluwalia y colaboradores ${ }^{29}$ reportaron que Streptococcus mutans se encontraron en mayor proporción en los niños con LPH que en los niños control ( $\mathrm{p}$ $<0,001)$.

Índice de Placa. Sundell y colaboradores ${ }^{18}$ también evaluaron este parámetro y como resultado se encontró que 57 personas del grupo control presentaban un bajo índice en contraste con solo 45 pertenecientes al grupo de LPH $(\mathrm{p}=0,02)$.

Por otro lado Chopra y colaboradores ${ }^{21}$ reportaron que en el grupo con LPH se encontró un promedio de índice de placa de 0,9 mientras que en el grupo control se halló uno de $0,7(\mathrm{p}=0,005)$.

Secreción salival. Sundell y colaboradores ${ }^{18}$ hallaron que 12 niños del grupo de LPH tenía baja secreción salival y solo 4 en el grupo control, sin embargo no se encontró significancia estadística $(p>0,05)$.

Maloclusión y lesiones en mucosas de la cavidad oral. Chopra y colaboradores ${ }^{21}$ fueron los únicos en evaluar este parámetro y se encontró un porcentaje de mordida abierta en el grupo LPH de 27,5\%, en comparación con el grupo control que fue de $3,1 \%(\mathrm{p}=0,003)$. Respecto a las lesiones en la mucosa presentaron más en los casos $(\mathrm{p}=0,042)$.

\section{DISCUSIÓN}

Esta investigación tuvo como objetivo evaluar la presencia de caries en los individuos con labio y paladar hendido mediante una revisión sistemática. Los resultados de este trabajo señalan que las personas con labio y paladar hendido son más susceptibles a presentar caries dental en comparación con quienes no presentan dicha condición. Este resultado se puede atribuir a que el labio y paladar hendido afectan directamente el desarrollo y crecimiento de las estructuras faciales $s^{30,31} \mathrm{y}$ trae como consecuencia al paciente, diversos problemas en la alimentación, la respiración nasal, la audición ${ }^{32}$, el crecimiento facial, el desarrollo 
dental, la fonación y la estética ${ }^{33}$, además de anomalías dentarias, adicionalmente, a nivel bucal se pueden generar hábitos de respiración bucal ${ }^{34}$, limpieza menos fácil de los dientes provocada por la disposición de estos, irregularidad de los dientes que produce un incremento en el acumulo de placa bacteriana y bacterias en la cavidad bucal de los niños con estas hendiduras bucales ${ }^{14,35,36}$.

Tabla 3. Prevalencia de caries reportada por los artículos

\begin{tabular}{|c|c|c|c|c|}
\hline \multirow{2}{*}{ Artículos } & \multirow{2}{*}{ Edad } & \multicolumn{2}{|c|}{ Participantes } & \multirow{2}{*}{ Valor $\mathbf{p}$} \\
\hline & & Casos n (\%) & Controles n (\%) & \\
\hline \multirow[t]{2}{*}{ Sundell y cols. ${ }^{18}$} & 5 años & 36,0 & 18,0 & $<0,05$ \\
\hline & 10 años & 47,0 & 38,0 & $>0,05$ \\
\hline Chopra y cols. ${ }^{21}$ & 4-6 años & 71,9 & 60,9 & 0,07 \\
\hline Pisek A y cols. ${ }^{22}$ & 10-14 años & 48,5 & 20,3 & $<0,01$ \\
\hline \multirow[t]{3}{*}{ Zhu WC y cols. ${ }^{26}$} & 3-5 años & 70,9 & 66,9 & 0,583 \\
\hline & 6-12 años & 82,4 & 69,9 & 0,019 \\
\hline & 13-25 años & 68,4 & 50 & 0,001 \\
\hline Mutarai T y cols. ${ }^{27}$ & 18 - 36 meses & 91,3 & 89,9 & 1,00 \\
\hline
\end{tabular}

Tabla 4. Otros parámetros evaluados por los artículos

\begin{tabular}{|c|c|c|c|}
\hline Artículos & Parámetros evaluados & Resultados & Conclusión \\
\hline Sundell y cols. ${ }^{18}$ & $\mathrm{DDE}$ & Pctes con Labio y paladar hendido: $\uparrow \mathrm{DDE}$ & $\mathrm{LPH}=\mathrm{DDE}$ \\
\hline Sundell y cols. ${ }^{19}$ & LB,ST, IP, SS & Pctes con Labio y paladar hendido: $\downarrow$ LB, $\uparrow S T, \uparrow I P, \downarrow S S$ & $\begin{array}{l}\mathrm{LPH}=\mathrm{LB}, \mathrm{ST}, \mathrm{IP} \\
\mathrm{LPH} \neq \mathrm{SS}\end{array}$ \\
\hline Chopra y cols. ${ }^{21}$ & IP, SG, MO, LM, DDE & Pctes con Labio y paladar hendido: $\uparrow \mathrm{IP}, \downarrow$ SG, $\uparrow \mathrm{MO}, \uparrow \mathrm{LM}$ & $\begin{array}{l}\mathrm{LPH}=\mathrm{MO}, \mathrm{IP}, \mathrm{SG} \\
\mathrm{LPH} \neq \mathrm{LM}, \mathrm{DDE}\end{array}$ \\
\hline Pisek 2014. ${ }^{22}$ & SG, IP,CV & Pctes con Labio y paladar hendido: $\uparrow I P, \downarrow$ SG & $\begin{array}{l}\mathrm{LPH}=\mathrm{IP}, \mathrm{SG} \\
\mathrm{LPH} \neq \mathrm{CV}\end{array}$ \\
\hline Freitas y cols. ${ }^{24}$ & SG, IP,AC & Pctes con Labio y paladar hendido: $\uparrow$ IP, $\downarrow$ SG, $\uparrow$ AC & $\begin{array}{l}\mathrm{LPH}=\mathrm{IP}, \mathrm{SG} \\
\mathrm{LPH} \neq \mathrm{AC}\end{array}$ \\
\hline Zhu y cols. ${ }^{26}$ & $\mathrm{AC}, \mathrm{HO}$ & Pctes con Labio y paladar hendido: $\uparrow \mathrm{HO}, \uparrow \mathrm{AC}$ & $\mathrm{LPH} \neq \mathrm{AC}$ \\
\hline Mutarai y cols. ${ }^{27}$ & $\mathrm{HO}, \mathrm{AC}$ & Pctes con Labio y paladar hendido: $\downarrow$ HO, $\uparrow$ AC, $\downarrow$ EF. & $\mathrm{LPH} \neq \mathrm{AC}, \mathrm{HO}$ \\
\hline Al-Wahadni y cols. ${ }^{28}$ & SG & Pctes con Labio y paladar hendido: $\downarrow$ SG & $\mathrm{LPH}=\mathrm{SG}$ \\
\hline Ahluwalia y cols. ${ }^{29}$ & LB,ST,AC, HO, SG & Pctes con Labio y paladar hendido: $\uparrow \mathrm{LB}, \uparrow S \mathrm{ST}, \downarrow \mathrm{AC}, \downarrow \mathrm{HO}, \downarrow \mathrm{SG}$ & $\begin{array}{l}\mathrm{LPH}=\mathrm{SG}, \mathrm{IP}, \mathrm{LB}, \mathrm{ST} \\
\mathrm{LPH} \neq \mathrm{AC} .\end{array}$ \\
\hline
\end{tabular}

DDE (Defecto desarrollo esmalte), LB (Lactobacilos), ST (Estreptococos), IP (Índice de placa), EF (Exposición al flúor), SS (Secreción salival), SG (Salud gingival), MO (Maloclusión), LM (Lesiones en mucosa), CV (Calidad de vida), AC (Alimentación cariogénica), HO (Higiene oral), = (Asociación), $\neq$ (sin asociación).

La presencia de diferentes anomalías relacionadas con esta patología se han descrito en diferentes investigaciones; autores como Rodríguez y colaboradores ${ }^{37}$ en el 2012 evaluaron mediante un estudio la prevalencia de alteraciones dentarias según la variación de tamaño, número y caries que presentaban los pacientes con LPH durante el período 2011-2012; ellos encontraron que respecto al número la agenesia fue el hallazgo más prevalente en estos pacientes con un $34 \%$ seguidos de dientes supernumerarios con un $23 \%$. La mayoría de estos eventos fueron más comunes en el sexo masculino ${ }^{37}$.
Asimismo, Antonarakis ${ }^{38}$, en su meta-análisis acerca de pacientes con labio y paladar hendido, expone varias causas por las cuales esta condición predispone a los individuos a desarrollar caries dental, como por ejemplo: la pérdida de elasticidad del labio producida por su reparación quirúrgica, la anatomía de la hendidura y el miedo al momento de realizar el cepillado de los dientes cerca del área de la hendidura, produciendo como consecuencia dificultades para conseguir una salud bucal óptima ${ }^{38}$. En contraste, Johnsen y Dixon ${ }^{39}$ en 1984 determinaron que el espacio 
restringido del arco generado por el subdesarrollo del maxilar puede causar un acceso limitado del cepillo de dientes al momento de realizar la limpieza bucal. Moura $^{40}$ en el 2013 realizó un estudio en el que evaluaba niños entre 6 y 36 meses de vida con al menos dos dientes erupcionados; gracias a su estudio determinó que el incisivo central superior, resultó ser el más afectado por la caries en estos pacientes.

Por ende los estudios publicados acerca de la presencia de caries dental en pacientes con fisuras orales $^{41-43}$ demuestran la importancia de mejorar la higiene oral, los hábitos alimenticios y suplementos de fluoruros $^{44}$ adecuados para, de esta manera, reducir la probabilidad de desarrollar caries dental ${ }^{45}$.

Hasslöf y Twetman ${ }^{46}$ hicieron una revisión sistemática acerca de la prevalencia de caries en niños con labio y paladar hendido, analizando estudios de casos y controles, en el cual pudieron concluir que los niños con LPH son más susceptibles a presentar caries dental con respecto a los niños que no tienen esta condición, aspecto que también puede confirmarse con la mayoría de artículos considerados para la presente investigación ${ }^{47}$.

Se puede destacar que en esta revisión sistemática se realizó una búsqueda exhaustiva de artículos en algunas de las bases con revistas de mayor impacto y citación en la literatura biomédica, también es importante aclarar que debido a la escasa evidencia observada en otros idiomas, cuando se hizo la búsqueda preliminar, se prefirió solo escoger los documentos publicados en inglés. Se sugiere que futuras investigaciones hagan una evaluación de la calidad de los artículos y su reporte con el fin de contribuir a la precisión en los relatos de estos artículos científicos.

\section{CONCLUSIÓN}

Finalmente, se concluye que la presencia del labio y paladar hendido pueden ser eventos que ubiquen en un riesgo mayor a los individuos que los presenten, y la investigación en odontología como también los clínicos necesitan desarrollar estrategias integrales donde se pueda garantizar una prevención personalizada a estos pacientes, para poder mitigar los aspectos ligados a su condición que desfavorecen su salud bu- cal y en algunos casos su estado sistémico. Indudablemente estas patologías pudiesen afectar directamente el estilo de vida de las personas que la padecen, ya que podrían presentar problemas estéticos y funcionales ${ }^{48}$, tales como trastornos en el lenguaje, dificultad al deglutir; respiración oral ${ }^{49}$. Por esta razón también es importante tratar esta anomalía, teniendo en cuenta sus respectivos cuidados ${ }^{50}$.

\section{BIBLIOGRAFÍA}

1. Cerón A, López A, Tirado C, Avendaño L. Prevalencia de caries en niños con labio y paladar hendido de la Fundación Clínica Noel, Medellín [Trabajo de grado]. Colombia: Universidad CES; 2011.

2. Serrano CA, Ruiz JM, Quiceno LF, Rodríguez MJ. Labio y/o paladar hendido: una revisión. Ustasalud. 2009;8(1):44-52.

3. Sotomayor J, Gutarra LA. Factores de riesgo en el embarazo para la presencia de fisuras labio máxilo palatinas. Odontol. Sanmarquina. 2004;8(1):17-24.

4. Corbo-Rodríguez MT, Marimón-Torres ME. Labio y paladar fisurados: Aspectos generales que se deben conocer en la atención primaria de salud. Revista Cubana de Medicina General Integral. 2001;17(4):379-85.

5. Long Jr RE, Hathaway R, Daskalogiannakis J, Mercado A, Russell K, Cohen $\mathrm{M}$ et al. The Americleft study: an inter-center study of treatment outcomes for patients with unilateral cleft lip and palate part 1. Principles and study design. Cleft Palate Craniofac J. 2011;48(3):239-43.

6. Wyszynski DF, Duffy DL, Beaty TH. Maternal cigarette smoking and oral clefts: a meta-analysis. Cleft Palate Craniofac J. 1997;34(3):206-10.

7. Cheng LL, Moor SL, Ho CT. Predisposing factors to dental caries in children with cleft lip and palate: a review and strategies for early prevention. Cleft Palate Craniofac J. 2007;44(1):67-72.

8. Shashni R, Goyal A, Gauba K, Utreja AK, Ray P, Jena AK. Comparison of risk indicators of dental caries in children with and without cleft lip and palate deformities. Contemp Clin Dent. 2015;6(1):58-62.

9. Wells M. Review suggests that cleft lip and palate patients have more caries. Evidence-Based Dentistry. 2014;15(3):79.

10. Hwang SJ, Beaty TH, Panny SR, Street NA, Joseph JM, Gordon S et al. Association Study of Transforming Grow- 
th Factor Alpha (TGFa) TaqI Polymorphismand Oral Clefts: Indication of Gene-Environment Interaction in a Population-based Sample of Infants with Birth Defects. American Journal of Epidemiology. 1995;141(7):629-36.

11. Shaw GM, Wasserman CR, Lammer EJ. Orofacial clefts, parental cigarette smoking, and transforming growth factor-alpha gene variants. Am J Hum Genet. 1996;58(3):551-61.

12. DeRoo LA, Gaudino JA, Edmonds LD. Orofacial cleft malformations: associations with maternal and infant characteristics in Washington State. Birth Defects Research: Clin A Mol Teratol. 2003;67(9):637-64.

13. Besseling S, Dubois L. The prevalence of caries in children with a cleft lip and/or palate in Southern Vietnam. Cleft Palate Craniofac J. 2004;41(6):629-32.

14. González MC, Gaona AM, Gamboa LF, Martignon S. Epidemiología de caries dental (ICDAS) en individuos colombianos con labio y paladar hendido. Universitas Odontológica. 2013; 32(68):125-32.

15. Fejerskov O, Kidd E. Dental caries: the disease and its clinical management. 3ra ed. Oxford: John Wiley \& Sons; 2009.

16. Moher D, Liberati A, Tetzlaff J, Altman DG, Prisma Group. Preferred reporting items for systematic reviews and meta-analyses: the PRISMA statement. PLoS medicine 2009;6(7):e1000097. doi: 10.1371/journal.pmed.1000097.

17. Microsoft Corporation Excel. 2015 United. States.

18. Sundell AL, Nilsson AK, Ullbro C, Twetman S, Marcusson A. Caries prevalence and enamel defects in 5- and 10-year-old children with cleft lip and/or palate: A case-control study. Acta Odontol Scand. 2015;74(2):90-5.

19. Sundell AL, Ullbro C, Marcusson A, Twetman S. Comparing caries risk profiles between 5 - and 10- year-old children with cleft lip and/or palate and non-cleft controls. BMC Oral Health. 2015;15(1):85 doi: 10.1186/ s12903-015-0067-x.

20. Kirchberg A, Makuch A, Hemprich A, Hirsch C. Dental caries in the primary dentition of german children with cleft lip, alveolus, and palate. Cleft Palate Craniofac J. 2014; 51(3):308-13.

21. Chopra A, Lakhanpal M, Rao NC, Gupta N, Vashisth S. Oral health in 4-6 years children with cleft lip/palate: A case control study. North Am y Med Sci. 2014;6(6):266-9.

22. Pisek A, Pitiphat W, Chowchuen B, Pradubwong S. Oral health status and oral impacts on quality of life in early adolescent cleft patients. J Med Assoc Thai. 2014;97(10):7-16.

23. King NM, Wong WL, Wong HM. Caries experience of chinese children with cleft lip and palate. Cleft Palate Craniofac J. 2013;50(4):448-55.

24. Freitas AB, de Barros LM, Fiorini JE, Boriollo MF, Moreira AN, Magalhaes CS. Caries experience in a sample of adolescents and young adults with cleft lip and palate in Brazil. Cleft Palate Craniofac J. 2013;50(2):187-91.

25. Tannure PN, Costa Mde C, Kuchler EC, Romanos HF, Granjeiro JM, Vieira AR. Caries experience in individuals with cleft lip and palate. Pediatr Dent. 2012;34(2):127-31.

26. Zhu WC, Xiao J, Liu Y, Wu J, Li JY. Caries experience in individuals with cleft lip and/or palate in China. Cleft Palate Craniofac J. 2010;47(1):43-7.

27. Mutarai T, Ritthagol W, Hunsrisakhun J. Factors influencing early childhood caries of cleft lip and/or palate children aged 18 to 36 months in southern Thailand. Cleft Palate Craniofac J. 2008;45(5):468-72.

28. Al-Wahadni A, Alhaija EA, Al-Omari MA. Oral disease status of a sample of Jordanian people ages 10 to 28 with cleft lip and palate. Cleft Palate Craniofac J. 2005;42(3):304-8.

29. Ahluwalia M, Brailsford SR, Tarelli E, Gilbert SC, Clark DT, Barnard K et al. Dental caries, oral hygiene, and oral clearance in children with craniofacial disorders. J Dent Res. 2004;83(2):175-9.

30. Britton K, Welbury R. Dental caries prevalence in children with cleft lip/palate aged between 6 months and 6 years in the West of Scotland. Eur Arch Paediatr Dent. 2010;11(5):236-42.

31. Agredo AFL, Rincón APM, Borrero ÁMD. Caracterización de pacientes con fisuras labiopalatinas atendidos en el Hospital Universitario del Valle en el período 20022011. Universitas Odontológica. 2014;33(70):79-4.

32. Lucas VS, Gupta R, Ololade O, Gelbier M, Roberts GJ. Dental health indices and caries associated microflora in children with unilateral cleft lip and palate. Cleft Palate Craniofac J. 2000;37(5):447-52.

33. Arévalo M, Canacuán F, Echeverry J, Salazar C, Martínez C, Cienfuegos A. Identificación molecular y genotipificación de Streptococcus mutans de muestras de saliva de niños de Medellín, Colombia. Revista CES Odontología. 2014;27(2):47-60. 
34. Paul T, Brandt RS. Oral and dental health status of children with cleft lip and/or palate. Cleft Palate Craniofac J. 1998;35(4):329-32.

35. de la Teja-Ángeles E, Durán-Gutiérrez A, Espinosa-Victoria L, Ramírez-Mayans JA. Manifestaciones estomatológicas de los trastornos sistémicos más frecuentes en el Instituto Nacional de Pediatría. Revisión de la literatura y estadísticas del instituto. Acta Pediátrica de México. 2008;29(4):189-99.

36. Hurtado AM, Sánchez DM, García AM, Ortíz MA, Aguirre AF. Prevalencia de caries y alteraciones dentarias en niños con labio fisurado y paladar hendido de una fundación de Santiago de Cali. Revista de Estomatología. 2008;16(1):13-17.

37. Rodríguez MB, Gonzáles LGV. Labio y paladar hendido: tendencias actuales en el manejo exitoso. Archivos de Medicina (Manizales). 2012;12(1):107-19.

38. Antonarakis GS, Palaska PK, Herzog G. Caries prevalence in non-syndromic patients with cleft lip and/or palate: a meta-analysis. Caries Res. 2013;47(5):406-13.

39. Johnsen DC, Dixon M. Dental caries of primary incisors in children with cleft lip and palate. Cleft Palate J. 1984;21:104-9.

40. Moura AM, André M, Lopez MT, Dias RB. Prevalence of caries in Brazilian children with cleft lip and/or palate, aged 6 to 36 months. Braz Oral Res. 2013;27(4):336-41.

41. Petersen PE. The World Oral Health Report 2003: continuous improvement of oral health in the 21 st century-the approach of the WHO Global Oral Health Programme. Community Dent Oral Epidemiol. 2003;31(s1):3-24.
42. Besseling S, Dubois L. The prevalence of caries in children with a cleft lip and/or palate in Southern Vietnam. Cleft Palate Craniofac J. 2004;41(6):629-32.

43. Stec-Slonicz M, Szczepańska J, Hirschfelder U. Comparison of caries prevalence in two populations of cleft patients. Cleft Palate Craniofac J. 2007;44(5):532-37.

44. Sosa-Rosales MC. Evolución de la fluoruración como medida para prevenir la caries dental. Revista Cubana de Salud Pública. 2003;29(3):268-74.

45. Lages EMB, Marcos B, Pordeus IA. Oral health of individuals with cleft lip, cleft palate, or both. Cleft Palate Craniofac J. 2004;41(1):59-63.

46. Hasslöf $\mathrm{P}$, Twetman S. Caries prevalence in children with cleft lip and palate-a systematic review of case-control studies. Int J Paediatr Dent. 2007;17(5):313-9.

47. Pinto ECH, Pinto EG, Soares S, Oliveira TM, Fraga ALP, Almeida JMB et al. A Critical Review of Dental Caries in Individuals with Cleft Lip. Dent. 2013;4(4):272-5.

48. Hewson A, McNamara C, Foley T, Sandy J. Dental experience of cleft affected children in the west of Ireland. Int Dent J. 2001;51(2):73-6.

49. Budai M, Kocsis SG, Kokai E, Sagi I, Mari A. Caries, gingivitis and dental abnormalities in patients with cleft lip and palate. Fogorv Sz. 2001;94(5):197-9.

50. Chapple J, Nunn J. Chapple JR, Nunn JH. The oral health of children with clefts of the lip, palate, or both. Cleft Palate Craniofac J. 2001;38(5):525-8. 